\title{
Pyrrolizidine alkaloids from Echium vulgare in honey originate primarily from floral nectar
}

\section{Matteo A. Lucchetti ${ }^{1,2}$, Gaetan Glauser ${ }^{3}$, Verena Kilchenmann ${ }^{1}$, Arne Dübecke ${ }^{4}$, Gudrun Beckh ${ }^{4}$, Christophe Praz $^{2}$, Christina Kast ${ }^{1 *}$}

${ }^{1}$ Agroscope, Swiss Bee Research Centre, Schwarzenburgstrasse 161, 3003 Bern, Switzerland.

${ }^{2}$ Institute of Biology, Laboratory of Fundamental and Applied Research in Chemical Ecology (FARCE), University of Neuchâtel, Rue Emile-Argand 11, 2000 Neuchâtel, Switzerland.

${ }^{3}$ Neuchâtel Platform of Analytical Chemistry, University of Neuchâtel, Rue Emile-Argand 11, 2000 Neuchâtel, Switzerland.

${ }^{4}$ Quality Services International GmbH (QSI), Flughafendamm 9a, 28199 Bremen, Germany.

*Corresponding author: Email: christina.kast@agroscope.admin.ch

Table S1: UHPLC-HRMS retention and mass characteristics (MS/MS ions) of Echium-type PAs.

\begin{tabular}{|c|c|c|c|c|c|c|}
\hline Peak $n^{\circ}$ & Compound Name & RT (min) & Chemical formula & $\begin{array}{c}{\left[\mathrm{M}+\mathrm{H}^{]+}\right.} \\
\text {experimental }\end{array}$ & $\begin{array}{c}{[\mathrm{M}+\mathrm{H}]^{+}} \\
\text {calculated }\end{array}$ & $\begin{array}{l}\text { Error } \\
\text { (mDa) }\end{array}$ \\
\hline 1 & Echimidine & 2.28 & $\mathrm{C} 2 \mathrm{OH} 31 \mathrm{NO} 7$ & 398.2184 & 398.2179 & 0.5 \\
\hline \multirow[t]{2}{*}{2} & Echimidine- $\mathrm{N}$-oxide & 2.27 & $\mathrm{C} 2 \mathrm{OH} 31 \mathrm{NO} 8$ & 414.2126 & 414.2128 & -0.2 \\
\hline & Vulgarine & 2.33 & $\mathrm{C} 2 \mathrm{OH} 31 \mathrm{NO} 7$ & 398.2174 & 398.2179 & -0.5 \\
\hline \multirow[t]{2}{*}{3} & Vulgarine-N-oxide & 2.43 & $\mathrm{C} 2 \mathrm{OH} 31 \mathrm{NO} 8$ & 414.2133 & 414.2128 & 0.5 \\
\hline & Acetylechimidine & 2.74 & $\mathrm{C} 22 \mathrm{H} 33 \mathrm{NO} 8$ & 440.2282 & 440.2284 & -0.2 \\
\hline \multirow[t]{2}{*}{4} & Acetylechimidine- $\mathrm{N}$-oxide & 2.71 & C22H33NO9 & 456.2234 & 456.2234 & 0.0 \\
\hline & Acetylvulgarine & 2.88 & $\mathrm{C} 22 \mathrm{H} 33 \mathrm{NO} 8$ & 440.2285 & 440.2284 & 0.1 \\
\hline \multirow[t]{2}{*}{5} & Acetylvulgarine- $\mathrm{N}$-oxide & 2.87 & $\mathrm{C} 22 \mathrm{H} 33 \mathrm{NO} 09$ & 456.2243 & 456.2234 & 0.9 \\
\hline & Echivulgarine & 3.81 & $\mathrm{C} 25 \mathrm{H} 37 \mathrm{NO} 8$ & 480.2575 & 480.2575 & 0.0 \\
\hline 6 & Echivulgarine- $\mathrm{N}$-oxide & 3.83 & C25H37NO9 & 496.3402 & 496.3405 & -0.3 \\
\hline
\end{tabular}




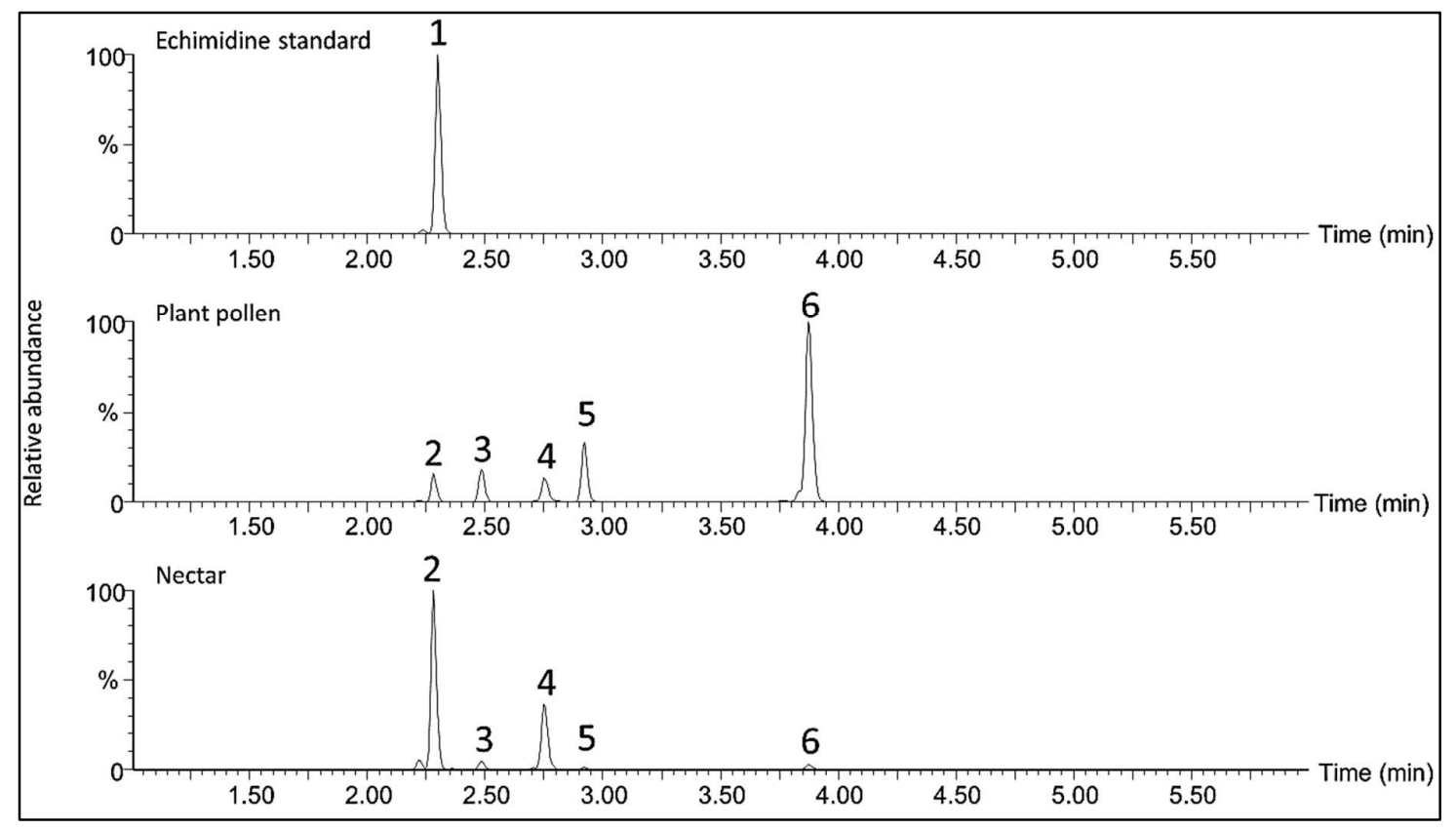

Figure S1: UHPLC-HRMS chromatograms of echimidine standard (1), PAs in pollen and nectar from Echium vulgare: echimidine-N-oxide (2), vulgarine- $\mathrm{N}$-oxide (3), acetylechimidine$\mathrm{N}$-oxide (4), acetylvulgarine- $\mathrm{N}$-oxide (5), echivulgarine- $\mathrm{N}$-oxide (6). Peak numbers also refer to Table S1.

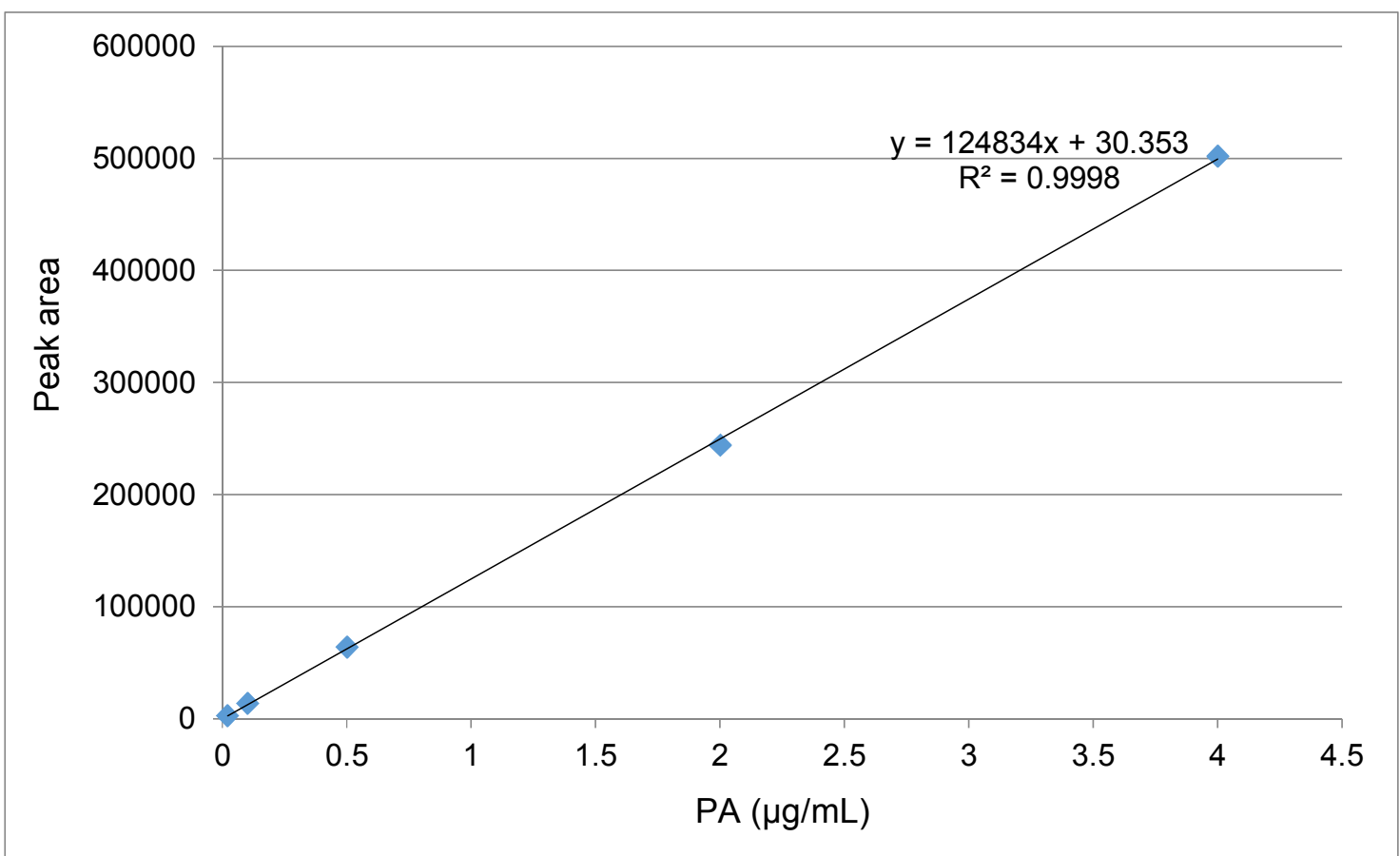

Figure S2: Linear range of echimidine standard in UHPLC-HRMS. Linearity was achieved from 0.005 to $4 \mu \mathrm{g} / \mathrm{mL}$. 